\title{
Morphological Control for Highly Efficient Inverted Polymer Solar Cells Via the Backbone Design of Cathode Interlayer Materials
}

\author{
Wenjun Zhang, Yulei Wu, Qinye Bao, Feng Gao and Junfeng Fang
}

\section{Linköping University Post Print}

\section{Tweet}

N.B.: When citing this work, cite the original article.

Original Publication:

Wenjun Zhang, Yulei Wu, Qinye Bao, Feng Gao and Junfeng Fang, Morphological Control for Highly Efficient Inverted Polymer Solar Cells Via the Backbone Design of Cathode Interlayer Materials, 2014, ADVANCED ENERGY MATERIALS, (4), 12, 1400359.

http://dx.doi.org/10.1002/aenm.201400359

Copyright: Wiley-VCH Verlag http://www.wiley-vch.de/publish/en/

Postprint available at: Linköping University Electronic Press http://urn.kb.se/resolve?urn=urn:nbn:se:liu:diva-110969 


\section{WILEY-VCH}

DOI: $10.1002 /(($ please add manuscript number $))$

Article type: Communication

\section{Morphological Control for Highly Efficient Inverted Polymer Solar Cells via Backbone Design of Cathode Interlayer Materials}

Wenjun Zhang, Yulei Wu, Qinye Bao, Feng Gao*, and Junfeng Fang*

((Optional Dedication))

Dr. W. Zhang, Mr. Y. Wu, Prof. J. Fang

Ningbo Institute of Materials Technology and Engineering, Chinese Academy of Sciences, Ningbo, 315201, China

E-mail: fangjf@nimte.ac.cn

Mr. Q. Bao, Dr. F. Gao

Biomolecular and Organic Electronics, IFM and Center of Organic Electronics, Linköping University, Linköping SE-581 83, Sweden

E-mail: fenga@ifm.liu.se

Keywords: organic solar cells, morphologies, structure-property relationships, cathode interlayers

Bulk-heterojunction (BHJ) polymer solar cells (PSCs), a blend of conjugated polymers and fullerene derivatives, are a promising candidate for next-generation low-cost energy conversion devices. ${ }^{[1]}$ In recent years, it has been recognized that the inverted device structure, where the modified indium tin oxide (ITO) is used as the cathode, is advantageous over the conventional device structure. ${ }^{[2]}$ Inverted devices have demonstrated much better device stability since they avoid the acid poly (3,4-ethylenedioxythiophene):(polystyrene sulfonic acid) (PEDOT:PSS) and the easily-oxidized low work function cathode metals. ${ }^{[3]}$ In addition, the inverted structure also takes advantage of the vertical composition gradient in the active layer, with fullerene-rich phase close to the cathode and polymer-rich phase close to the anode. ${ }^{[4]}$ As a result, the record efficiency $(9.2 \%)$ of single-junction PSCs has been achieved in the inverted device. ${ }^{[5]}$

The key issue in inverted PSCs is the development of interlayers, which make the ITO suitable to work as the cathode. ${ }^{[6]}$ The interfacial materials can be grouped into two categories, i.e. inorganic and organic materials. Although inorganic interfacial materials also give decent 


\section{WILEY-VCH}

device performance, ${ }^{[7]}$ organic materials are believed to have better compatibility with the active layer, assisting morphology optimization and charge extraction. ${ }^{[8]}$ For example, in the single-junction PSC with the record efficiency, an organic interlayer was employed. ${ }^{[5]}$ In these organic interfacial materials, the interface dipole introduced by the pendant group plays a critical role in adjusting the work function of the ITO and enhancing the electron collection from the acceptor to ITO ${ }^{[9]}$ In principle, the conjugated backbones of these interlayers are also important in interfacial modification, since they affect the charge transport, energy gaps, hydrophobicity, etc. ${ }^{[10]}$ In spite of intensive research in this area, little is known about the effect of the backbones of the interfacial materials on the performance of inverted PSCs. ${ }^{[1]}$

In this communication, we developed two alcohol-soluble organic small molecules as interlayers for highly efficient inverted PSCs. By modifying the conjugated backbones while fixing the polar pendant group, we aim to investigate the relationship between the backbones of cathode interlayer materials and device performance. Simply by adding two thiophene units to the backbone of the interfacial materials, we can increase the device power conversion efficiency from less than $8.0 \%$ to $9.2 \%$. We find that the difference in the device performance is caused by the difference in the morphology of the active layer, which can be significantly influenced by the conjugated backbone of the cathode interlayer materials.

The structures of the two alcohol-soluble small molecules are shown in Scheme 1. FBF$\mathrm{N}$ and FTBTF-N possess similar structure, with a conjugated backbone and a side chain pendant N,N-dimethyl amino group. Both of them were synthesized through Suzuki coupling reaction with good yields over $60 \%$. Fluorene units are linked by benzo[ $[c][1,2,5]$ thiadiazole and bisthiophenyl-benzo $[c][1,2,5]$ thiadiazole in FBF-N and FTBTF-N, respectively. Owing to the polar N,N-dimethyl amino end group, both compounds have a good solubility in polar solvents, such as methanol and N,N-dimethylformamide.

Ultraviolet-visible (UV-vis) absorption spectra and cyclic voltammogram (CV) of FBF$\mathrm{N}$ and FTBTF-N are shown in Figure S1, and relevant data are listed in Table S1. Films were 


\section{WILEY-VCH}

prepared by spin-casting from methanol solutions on quartz substrates. In solution, the absorption maximum for FBF-N is located at $317 \mathrm{~nm}$ and $408 \mathrm{~nm}$, while that for FTBTF-N is at $363 \mathrm{~nm}$ and $502 \mathrm{~nm}$, respectively. The longer wavelength absorption bands for FBF-N and FTBTF-N can be assigned to intramolecular charge transfer (ICT) transition. ${ }^{[1 \mathrm{c}]}$ Obviously, there is a significant red shift from FBF-N to FTBTF-N. The tendency is in accordance with the structure changes between the backbones of the two compounds, as FTBTF-N possesses a more intense ICT transition from the thiophene unit to the benzothiadiazole unit. The highest occupied molecular orbital (HOMO) values for FBF-N and FTBTF-N are estimated to be 5.60 and $-5.23 \mathrm{eV}$, and the calculated lowest unoccupied molecular orbital (LUMO) values are -2.99 and $-3.33 \mathrm{eV}$. Accordingly, the electrochemical band gaps $\left(\mathrm{E}_{\mathrm{g}}^{\mathrm{CV}}\right)$ of FBF-N and FTBTF-N are 2.61, and $1.90 \mathrm{eV}$ respectively, which is consistent with the optical band gaps $\left(\mathrm{E}_{\mathrm{g}}^{\mathrm{opt}}\right)$.

To examine the performance of inverted PSCs with the two compounds as cathode interlayers and investigate the influence of backbones on the device performance, a highly efficient photovoltaic blend of poly[4,8-bis(2-ethylhexyloxyl)benzo[1,2-b:4,5- $\left.b^{\prime}\right]$ dithiophene2,6-diyl-alt-ethylhexyl-3-uorothithieno[3,4-b]thiophene-2-carboxylate-4,6-diyl] (PTB7) and (6,6)-phenyl- $\mathrm{C}_{71}$-butyric acid methyl ester $\left(\mathrm{PC}_{71} \mathrm{BM}\right)$ blend was chosen as the photoactive layer in our inverted PSCs. ${ }^{[1 \mathrm{~d}]}$ The device configuration was ITO/cathode interlayer/PTB7: $\mathrm{PC}_{71} \mathrm{BM} / \mathrm{MoO}_{3} / \mathrm{Al}$. The cathode interlayer was prepared by spin-coating the methanol solution of each compound on the ITO substrate. Control devices without the cathode interlayer were also fabricated.

The current density-voltage $(J-V)$ characteristics of the optimized devices under illumination of AM 1.5G, $100 \mathrm{~mW} \mathrm{~cm}^{-2}$ and at dark are shown in Figure 1, with the device parameters summarized in Table 1. Obviously, both devices with FBF-N or FTBTF-N as the cathode interlayer show high PCEs, which indicate that both FBF-N and FTBTF-N are effective materials for cathode interfacial modification. With FBF-N as the cathode interlayer, 


\section{WILEY-VCH}

the inverted PSCs exhibited an average PCE of $7.85 \%$, a $V_{o c}$ of $0.75 \mathrm{~V}$, a $J_{s c}$ of $15.67 \mathrm{~mA} \mathrm{~cm}^{-2}$, and an $F F$ of $66.62 \%$. With FTBTF-N as the cathode interlayer, the inverted PSCs exhibited an average PCE of $8.93 \%$, a $V_{o c}$ of $0.74 \mathrm{~V}$, a $J_{s c}$ of $17.30 \mathrm{~mA} \mathrm{~cm} \mathrm{~cm}^{-2}$, and an $F F$ of $69.85 \%$. The small difference in $V_{o c}$ between these two devices $(0.75 \mathrm{~V}$ for the FBF-N device and $0.74 \mathrm{~V}$ for the FTBTF-N device) is attributed to the experimental error. The best FTBTF-N device showed a PCE value as high as $9.22 \%$, which is among the highest PCEs for single-juncton BHJ PSCs. ${ }^{[5]}$ Compared to the devices with FBF-N as the cathode interlayer, the improvement in the performance of the FTBTF-N device mainly arises from higher $J_{s c}$ and FF.

One of the direct reasons that could result in the difference in $J_{s c}$ is different exciton generation rate. Since the HOMO/LUMO levels of PTB7 lie between those of the interlayer materials, ${ }^{[1 \mathrm{~d}]}$ the exciton generation in the active layer could be potentially affected by energy transfer from the interlayer materials to the active layer materials. Therefore, we normalized the external quantum efficiency (EQE) spectra at $680 \mathrm{~nm}$, where neither FTBTF-N nor FBF$\mathrm{N}$ absorbs. As shown in Figure S2a, indeed, the FTBTF-N device demonstrates slightly higher EQE values in the wavelength regime where FTBTF-N absorbs, indicating possible energy transfer from the interlayers to PTB7. In order to examine how strong this effect could be, we compared the absorption spectrum of the active layer on the FBF-N film with that on FTBTF-N. As shown in Figure S2b, consistent with EQE spectra, slightly stronger absorption of the active layer on the FTBTF-N film is observed in the regime where FTBTF$\mathrm{N}$ absorbs. However, the difference in absorption is small. A rough estimation by integrating the absorption spectra over the solar spectrum shows that the small difference in absorption could only result in less than $1 \%$ difference in exciton generation, which could not explain over $10 \%$ difference in photocurrent generation. ${ }^{[12]}$ Therefore, the difference in $J_{s c}$ of the two devices is supposed to result from subsequent charge generation and recombination processes, rather than from exciton generation. 


\section{WILEY-VCH}

The work function of the ITO substrates with and without the cathode interlayer was measured by ultraviolet photoelectron spectroscopy (Figure S3). The work function was measured to be $4.40 \mathrm{eV}$ for a bare ITO substrate while it was $4.10 \mathrm{eV}$ and $4.06 \mathrm{eV}$ for FBF-N and FTBTF-N coated ITO substrates. The work function of ITO can be decreased by $\sim 0.3 \mathrm{eV}$ via the introduction of a thin layer of FBF-N or FTBTF-N. The lower work function of the modified ITO substrate increases the built-in potential and benefits the electron collection, both of which are responsible for the enhancement of the device efficiency compared with the control device. ${ }^{[13]}$ The similar work function of FBF-N and FTBTF-N coated ITO is also consistent with similar $V_{o c}$ of the FBF-N devices and FTBTF-N devices.

Since the work functions of ITO/FBF-N and ITO/FTBTF-N substrates are similar, the higher $J_{s c}$ and $F F$ values of FTBTF-N devices could not be derived from the interface charge transport or extraction. Atomic force microscopy (AFM) was then carried out to examine the morphologies of the photoactive layers on an FBF-N or FTBTF-N surface. Figure 2 compares the surface morphologies of PTB7:PC ${ }_{71} \mathrm{BM}$ on FBF-N and FTBTF-N surfaces. Apparently, the photoactive layer deposited on the FTBTF-N surface exhibited lower roughness than that on the FBF-N surface. The root-mean-square (RMS) for PTB7:PC $71 \mathrm{BM}$ on the FBF-N surface and on the FTBTF-N surface is $2.87 \mathrm{~nm}$ and $0.98 \mathrm{~nm}$, respectively. In addition, the photoactive layer deposited on the FTBTF-N surface shows an obvious and homogeneous phase separation, which was not observed from the photoactive layer deposited on the FBF-N surface. Comparison of the AFM images indicates that the PTB7:PC ${ }_{71} \mathrm{BM}$ film deposited on the FTBTF-N film might have better phase separation and continuous interpenetrating networks compared with that on the FBF-N film. ${ }^{[1 \mathrm{e}]}$ It has been well acknowledged that continuous interpenetrating networks with proper domain size are essential for the efficient exciton separation and charge transport, which are important factors to improve the $J_{s c}$ and $F F$. Better morphology for the FTBTF-N devices is consistent with the higher $J_{s c}$ and $F F$ values compared with the FBF-N devices. ${ }^{[14]}$ 


\section{WILEY-VCH}

In order to have a deep insight of the higher $J_{s c}$ and $F F$ of FTBTF-N devices induced by photoactive morphology, highly sensitive electroluminescence (EL) spectra and Fouriertransform photocurrent spectroscopy (FTPS) measurements were carried out. EL and FTPS measurements probe the weak emission and absorption of charge-transfer (CT) states between the donor and the acceptor in PSCs. While AFM examines the morphology on the surface, EL and FTPS could provide morphology information of the whole active layer ${ }^{[15]}$ Figure 3a compares the EL spectra of the FBF-N device and the FTBTF-N device, which were measured using the same injection current and normalized at the peak position of the CT emission. The emission band around $820 \mathrm{~nm}$ and $1000 \mathrm{~nm}$ are assigned to pure PTB7 emission and the PTB7/PC ${ }_{71} \mathrm{BM}$ interfacial $\mathrm{CT}$ emission, respectively. The FBF-N device exhibited an obvious emission at $820 \mathrm{~nm}$, which was not observed in the FTBTF-N device. This means that the pure PTB7 polymer emission is more suppressed in the FTBTF-N device, indicating better phase separation and larger PTB7/PC $71 \mathrm{BM}$ interfacial area. This conclusion is consistent with higher photocurrent for the FTBTF-N device. The FTPS Spectra (Figure 3b) are normalized in the region of polymer absorption. There is an increase for the CT emission signal from the sample based on the FTBTF-N device, indicating better intermixing between the donor and the acceptor. ${ }^{[16]}$ This agrees well with the reduced domain size observed in AFM and decreased pure polymer EL in the FTBTF-N device. Thus it could be concluded that the increased $\mathrm{PTB} 7 / \mathrm{PC}_{71} \mathrm{BM}$ interfacial area contributes to the observed increase in $J_{s c}$ and $F F$.

In addition to the morphological variation, we also investigated the wetting properties of the cathode interlayers. Contact angle measurements were performed on both surfaces with drops of water. The water contact angle for the FBF-N surface is $38^{\circ}$, and it was increased to $69^{\circ}$ for the FTBTF-N surface (Figure S4). The larger contact angle of the ITO/FTBTF-N surface indicates that FTBTF-N is more hydrophobic and the photoactive solution will better spread out. ${ }^{[7 \mathrm{~b}]}$ The better wetting properties for PTB7:PC ${ }_{71} \mathrm{BM}$ solution could lead to a better 


\section{WILEY-VCH}

morphology and interpenetrating networks. Combining the AFM images and contact angle measurements, we conclude that the more effective phase separation of $\mathrm{PTB} 7 / \mathrm{PC}_{71} \mathrm{BM}$ deposited on FTBTF-N is induced by the more hydrophobic property of FTBTF-N than FBFN. Since FBF-N and FTBTF-N possess the same polar pendant, the different hydrophobic properties of them can be ascribed to the different backbones. The larger $\pi$-extended system of FTBTF-N could be attributed to the more hydrophobic property of FTBTF-N than FBFN. ${ }^{[17]}$ Previous researches about alcohol-soluble organic cathode interlayers have focused on how to reduce the work function of ITO. Here, our results suggest that not only the polar pendant group can help to reduce the work function of ITO, which will increase $V_{o c}$, but also the design of backbone can help to modify the hydrophobic property of interlayer, which will enhance $J_{s c}$ and $F F$.

In summary, two hydrophilic small molecules were synthesized and used as the cathode interlayer in inverted PSCs. Both of them exhibited efficient cathode interlayer modification, and with FTBTF-N as the cathode interlayer, a PCE value as high as $9.22 \%$ was achieved. Compared with FBF-N devices, FTBTF-N devices exhibited a higher $J_{s c}$ and $F F$. The higher $J_{s c}$ and $F F$ were attributed to the more hydrophobic property of FTBTF-N, leading to the formation of better morphology upon the photoactive layer deposition. The effect of the backbone structures of cathode interlayer materials on the performance of inverted PSCs was investigated. We found that the cathode interlayer could enhance not only $V_{o c}$ but also $J_{s c}$ and FF of inverted PSCs by structural design of polar pendant group and backbone. Our work boosts the understanding to the cathode interlayer of inverted PSCs and provides a new point of view to the development of novel cathode interlayer materials for inverted PSCs.

\section{Experimental Section}

Measurement and characterization: ${ }^{1} \mathrm{H}$ NMR and ${ }^{13} \mathrm{C}$ NMR spectra were recorded on a Bruker $400 \mathrm{MHz}$ AVANCE III with tetramethylsilane as an internal reference. Mass spectra 


\section{WILEY-VCH}

were obtained on a Finnigan LCQ mass spectrometer. UV-vis absorption spectra were recorded on a mapada UV-3300 spectrophotometer. Cyclic voltammetry (CV) was carried out on a CHI600D electrochemical workstation with a platinum working electrode of $2 \mathrm{~mm}$ diameter and a platinum wire counter electrode at a scan rate of $50 \mathrm{mV} \mathrm{s}^{-1}$ against a saturated calomel electrode (SCE) reference electrode with an argon saturated anhydrous solution of 0.1 $\mathrm{mol} / \mathrm{L}$ tetrabutylammonium hexafluorophosphate $\left(\mathrm{Bu}_{4} \mathrm{NPF}_{6}\right)$ in acetonitrile. The film for electrochemical measurements was coated from its dichloromethane solution. The tappingmode AFM images were obtained by using a scanning probe microscope (Dimension3100V). Contact angle measurements were performed on a video-based optical contact angle measuring instrument (OCA20, Dataphysics Corp.). EL spectra were obtained with a Shamrock SR-303i spectrograph from Andor Tech., coupled to a Newton EM-CCD Si array detector. The highly sensitive FTPS were measured using a Vertex 70 from Bruker optics.

Materials: All chemicals were purchased from commercial sources and used without further purification unless otherwise noted. Tetrahydrofuran (THF) and toluene were purified by distillation from Na-K under dry argon. Electron donor material PTB7 and electron $\mathrm{PC}_{71} \mathrm{BM}$ were purchased from 1-material chemscitech and American Dye Source Incorporated, respectively.

Device fabrication and characterization: Indium tin oxide (ITO)-coated glass substrates were cleaned by sonication in detergent, deionized water, acetone and isopropyl alcohol and dried in a nitrogen stream, followed by a UV ozone treatment. Then the cathode interlayer was deposited by spin-casting from FBF-N or FTBTF-N solution in methanol. The optimized concentrations for spin casting FBF-N and FTBTF-N cathode interlayer solutions were found to be at $8 \mathrm{mg} / \mathrm{mL}$. Subsequently, a photoactive layer of PTB7/PC ${ }_{71} \mathrm{BM}(10: 15$, w/w) was spincoated from chlorobenzene solution with $3 \%$ (w) 1,8-diiodooctane, which results in an active layer with the thickness of $90 \pm 10 \mathrm{~nm}$. Finally, a $10 \mathrm{~nm} \mathrm{MoO}$ layer and a $160 \mathrm{~nm}$ Al layer 


\section{WILEY-VCH}

were evaporated in sequence through a shadow mask to define the active area of the devices $\left(0.04 \mathrm{~cm}^{2}\right)$ and formed a top anode.

The anode thickness was monitored upon deposition by using a crystal thickness monitor (Sycon). Device fabrication was carried out in a nitrogen atmosphere dry glovebox. The current-voltage $(J-V)$ characteristics were recorded with a Keithley 236 source meter. The spectral response was measured with a commercial 40 photomodulation spectroscopy setup (Oriel). A calibrated Si photodiode was used to determine the photosensitivity. PCE was measured under an AM1.5G solar simulator. The power of the sun simulation was calibrated before the measurement using a standard silicon solar cell.

\section{Supporting Information}

Supporting Information is available from the Wiley Online Library or from the author.

\section{Acknowledgements}

The authors thank Dr. M. Lu for helpful discussions. Project 51273208 was supported by National Natural Science Foundation of China. The work was also supported by China Postdoctoral Science Foundation funded project, Ningbo Natural Science Foundation of China (2013A610132), Zhejiang Postdoctoral Science Foundation (BSH1301023). F.G. acknowledges the financial support of the European Commission under a Marie Curie IntraEuropean Fellowship for Career Development.

Received: ((will be filled in by the editorial staff))

Revised: ((will be filled in by the editorial staff)) Published online: ((will be filled in by the editorial staff))

[1] a) T.-Y. Chu, J. Lu, S. Beaupré, Y. Zhang, J.-R. m. Pouliot, S. Wakim, J. Zhou, M. Leclerc, Z. Li, J. Ding, Y. Tao, J. Am. Chem. Soc. 2011, 133, 4250; b) J. Chen, Y. Cao, Acc. Chem. Res. 2009, 42, 1709; c) Y. J. Cheng, S. H. Yang, C. S. Hsu, Chem. Rev. 2009, 109, 5868; d) Y. Liang, Z. Xu, J. Xia, S.-T. Tsai, Y. Wu, G. Li, C. Ray, L. Yu, Adv. Mater. 2010, 22, E135; e) B. C. Thompson, J. M. J. Fréchet, Angew. Chem. Int. Ed. 2008, 47, 58. 


\section{WILEY-VCH}

[2] a) M. S. White, D. C. Olson, S. E. Shaheen, N. Kopidakis, D. S. Ginley, Appl. Phys. Lett. 2006, 89, 143517; b) G. Li, C.-W. Chu, V. Shrotriya, J. Huang, Y. Yang, Appl. Phys. Lett. 2006, 88, 253503; c) A. K. K. Kyaw, D. H. Wang, V. Gupta, J. Zhang, S. Chand, G. C. Bazan, A. J. Heeger, Adv. Mater. 2013, 25, 2397; d) L.-M. Chen, Z. Hong, G. Li, Y. Yang, Adv. Mater. 2009, 21, 1434.

[3] a) M. P. d. Jong, L. J. v. IJzendoorn, M. J. A. d. Voigt, Appl. Phys. Lett. 2000, 77, 2255; b) K. Kawano, R. Pacios, D. Poplavskyy, J. Nelson, D. D. C. Bradley, J. R. Durrant, Sol. Energy Mater. Sol. Cells 2006, 90, 3520; c) M. Jørgensen, K. Norrman, F. C. Krebs, Sol. Energy Mater. Sol. Cells 2008, 92, 686.

[4] a) M. Campoy-Quiles, T. Ferenczi, T. Agostinelli, P. G. Etchegoin, Y. Kim, T. D. Anthopoulos, P. N. Stavrinou, D. D. C. Bradley, J. Nelson, Nat. Mater. 2008, 7, 158; b) Y. Yao, J. Hou, Z. Xu, G. Li, Y. Yang, Adv. Funct. Mater. 2008, 18, 1783; c) Z. Xu, L.-M. Chen, G. Yang, C.-H. Huang, J. Hou, Y. Wu, G. Li, C.-S. Hsu, Y. Yang, Adv. Funct. Mater. 2009, $19,1227$.

[5] Z. He, C. Zhong, S. Su, M. Xu, H. Wu, Y. Cao, Nat. Photonics 2012, 6, 591.

[6] a) S. K. Hau, H.-L. Yip, O. Acton, N. S. Baek, H. Ma, A. K. Y. Jen, J. Mater. Chem. 2008, 18, 5113; b) H.-H. Liao, L.-M. Chen, Z. Xu, G. Li, Y. Yang, Appl. Phys. Lett. 2008, 92, 173303; c) D.-Y. Chung, D.-S. Leem, D. D. C. Bradley, A. J. Campbell, Appl. Phys. Lett. 2011, 98, 103306; d) S. K. Hau, H.-L. Yip, N. S. Baek, J. Zou, K. O'Malley, A. K.-Y. Jen, Appl. Phys. Lett. 2008, 92, 253301.

[7] a) Y. Sun, J. H. Seo, C. J. Takacs, J. Seifter, A. J. Heeger, Adv. Mater. 2011, 23, 1679; b) Z. a. Tan, W. Zhang, Z. Zhang, D. Qian, Y. Huang, J. Hou, Y. Li, Adv. Mater. 2012, 24, 1476; c) S. Trost, K. Zilberberg, A. Behrendt, T. Riedl, J. Mater. Chem. 2012, 22, 16224.

[8] a) J. Sun, Y. Zhu, X. Xu, L. Lan, L. Zhang, P. Cai, J. Chen, J. Peng, Y. Cao, J. Phys. Chem. C 2012, 116, 14188; b) S.-I. Na, T.-S. Kim, S.-H. Oh, J. Kim, S.-S. Kim, D.-Y. Kim, Appl. Phys. Lett. 2010, 97, 223305; c) H. Kang, S. Hong, J. Lee, K. Lee, Adv. Mater. 2012, 24, 


\section{WILEY-VCH}

3005; d) S. Liu, K. Zhang, J. Lu, J. Zhang, H.-L. Yip, F. Huang, Y. Cao, J. Am. Chem. Soc. 2013, 135, 15326; e) K. Zilberberg, A. Behrendt, M. Kraft, U. Scherf, T. Riedl, Org. Electron. 2013, 14,951 .

[9] a) F. Zhang, M. Ceder, O. Inganäs, Adv. Mater. 2007, 19, 1835; b) Z. He, C. Zhong, X. Huang, W.-Y. Wong, H. Wu, L. Chen, S. Su, Y. Cao, Adv. Mater. 2011, 23, 4636.

[10] C. V. Hoven, A. Garcia, G. C. Bazan, T.-Q. Nguyen, Adv. Mater. 2008, 20, 3793.

[11] C. Duan, K. Zhang, X. Guan, C. Zhong, H. Xie, F. Huang, J. Chen, J. Peng, Y. Cao, Chem. Sci. 2013, 4, 1298.

[12] K. Yao, C. Liu, Y. Chen, L. Chen, F. Li, K. Liu, R. Sun, P. Wang, C. Yang, J. Mater. Chem. 2012, 22, 7342.

[13] a) C. He, C. Zhong, H. Wu, R. Yang, W. Yang, F. Huang, G. C. Bazan, Y. Cao, J. Mater. Chem. 2010, 20, 2617; b) J. Luo, H. Wu, C. He, A. Li, W. Yang, Y. Cao, Appl. Phys. Lett. 2009, 95, 043301.

[14] L. Dou, J. Gao, E. Richard, J. You, C.-C. Chen, K. C. Cha, Y. He, G. Li, Y. Yang, J. Am. Chem. Soc. 2012, 134, 10071.

[15] K. Tvingstedt, K. Vandewal, A. Gadisa, F. Zhang, J. Manca, O. Inganäs, J. Am. Chem. Soc. 2009, 131, 11819.

[16] Z. Tang, L. M. Andersson, Z. George, K. Vandewal, K. Tvingstedt, P. Heriksson, R. Kroon, M. R. Andersson, O. Inganäs, Adv. Mater. 2012, 24, 554.

[17] M. Wang, S.-J. Moon, D. Zhou, F. Le Formal, N.-L. Cevey-Ha, R. Humphry-Baker, C. Grätzel, P. Wang, S. M. Zakeeruddin, M. Grätzel, Adv. Funct. Mater. 2010, 20, 1821. 


\section{WILEY-VCH}

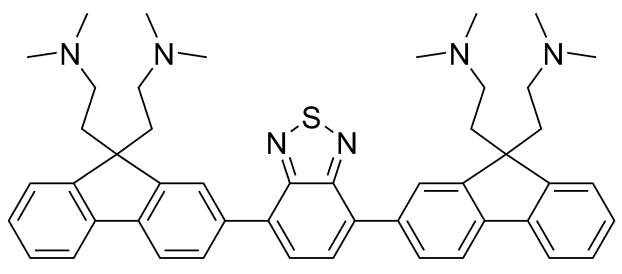

FBF-N

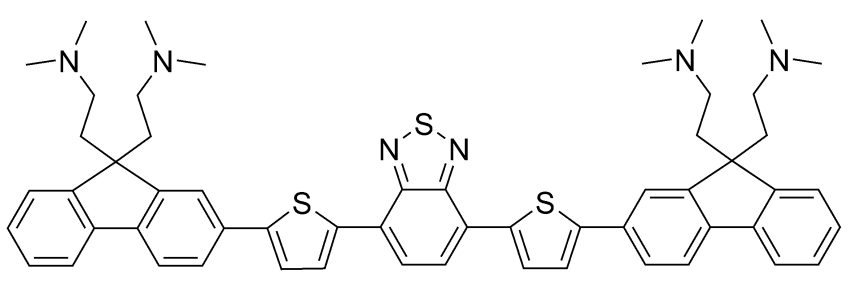

FTBTF-N

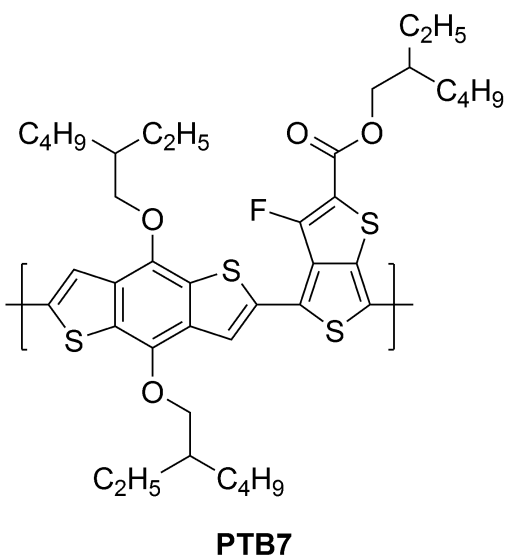

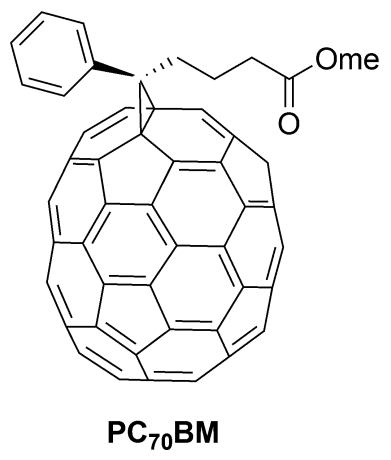

Scheme 1. Molecule structures of FBF-N, FTBTF-N and photoactive materials in our study.

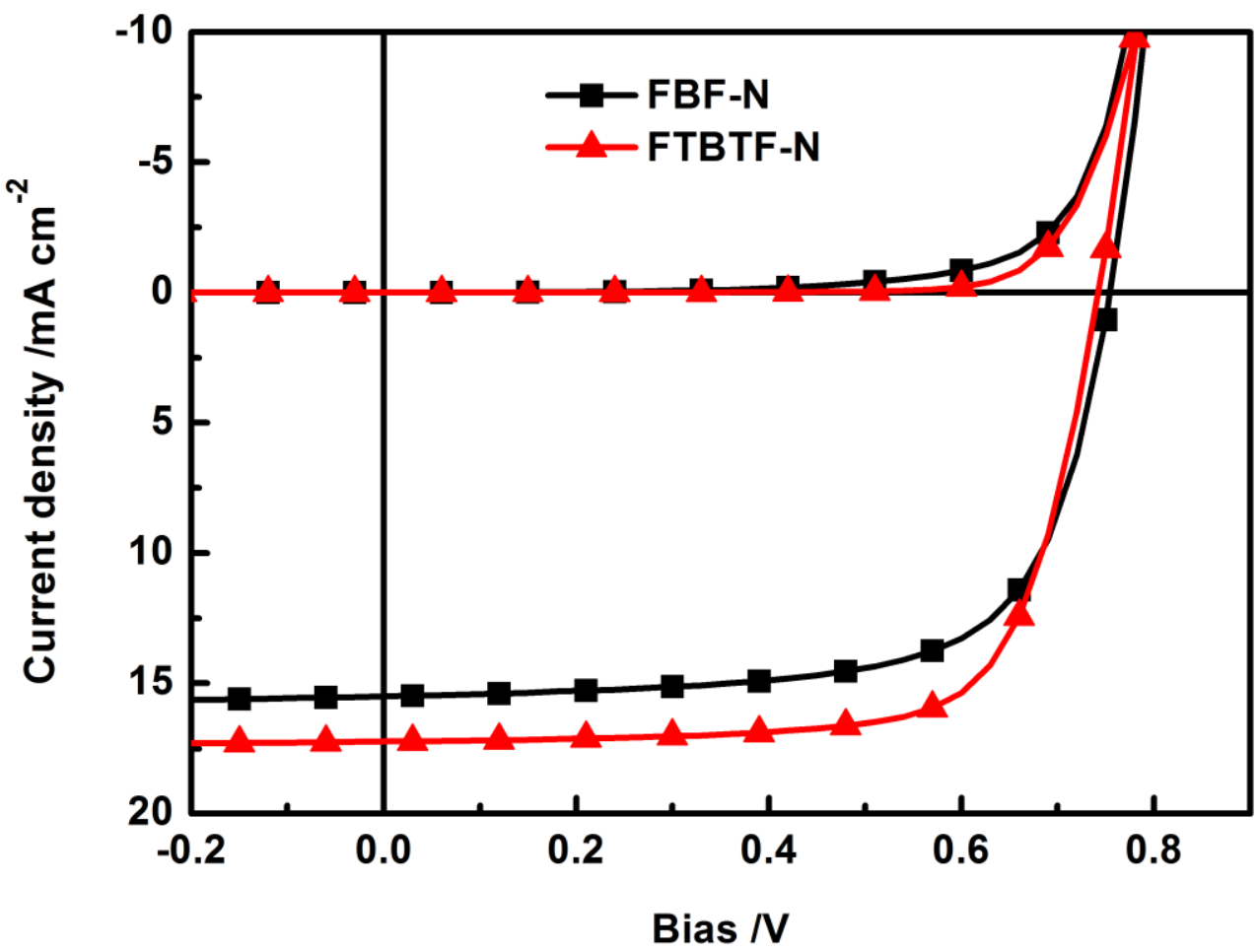

Figure 1. $J-V$ characteristics of devices with FBF-N and FTBTF-N interlayers in the dark and under AM $1.5 \mathrm{G}$ illumination of $100 \mathrm{~mW} \mathrm{~cm}^{-2}$. 


\section{WILEY-VCH}

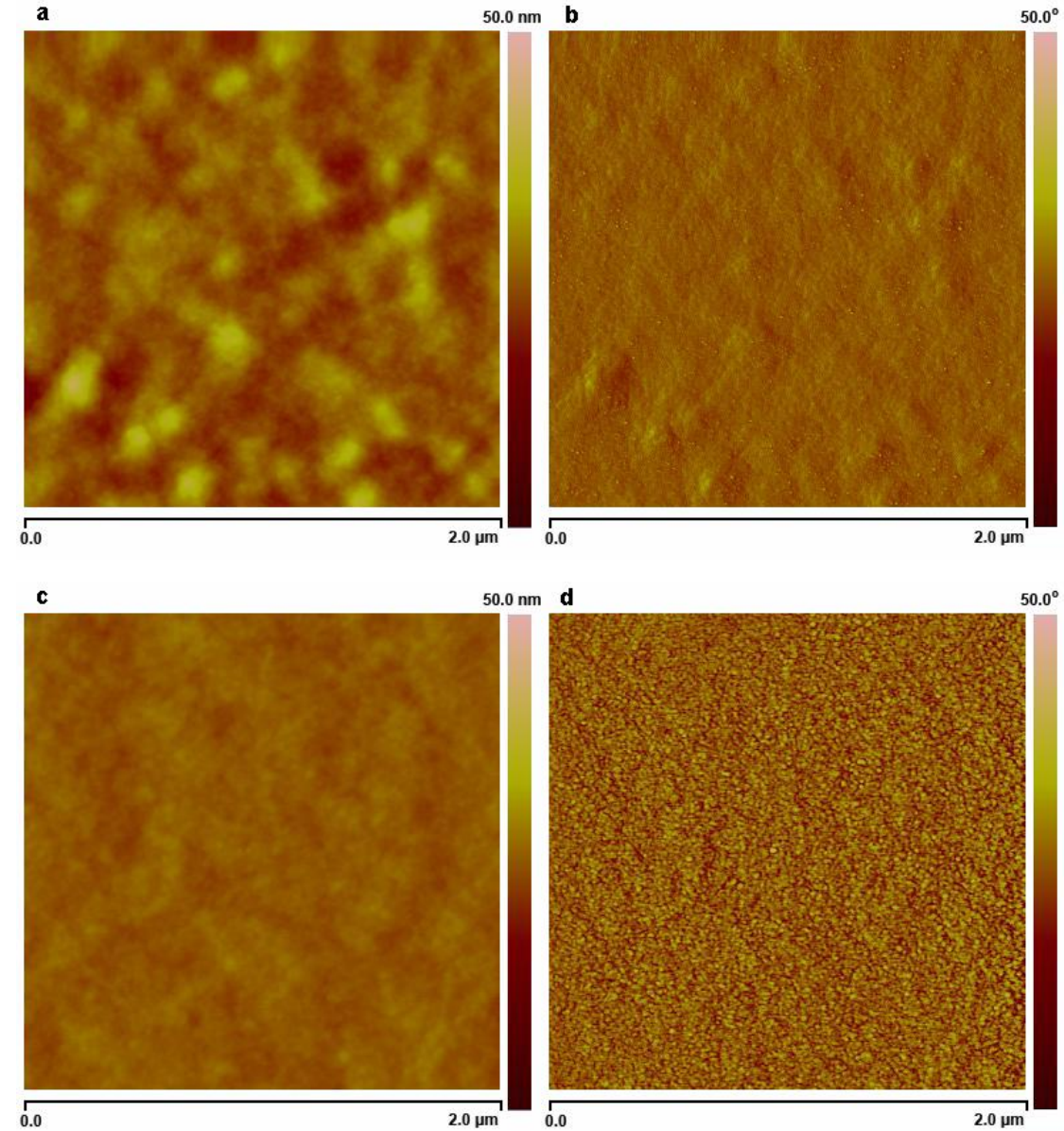

Figure 2. Tapping-mode AFM height (a, c) and phase (b, d) images of the PTB7:PC 71 BM film on the top of FBF-N (a, b) and FTBTF-N (c, d) film. 


\section{WILEY-VCH}
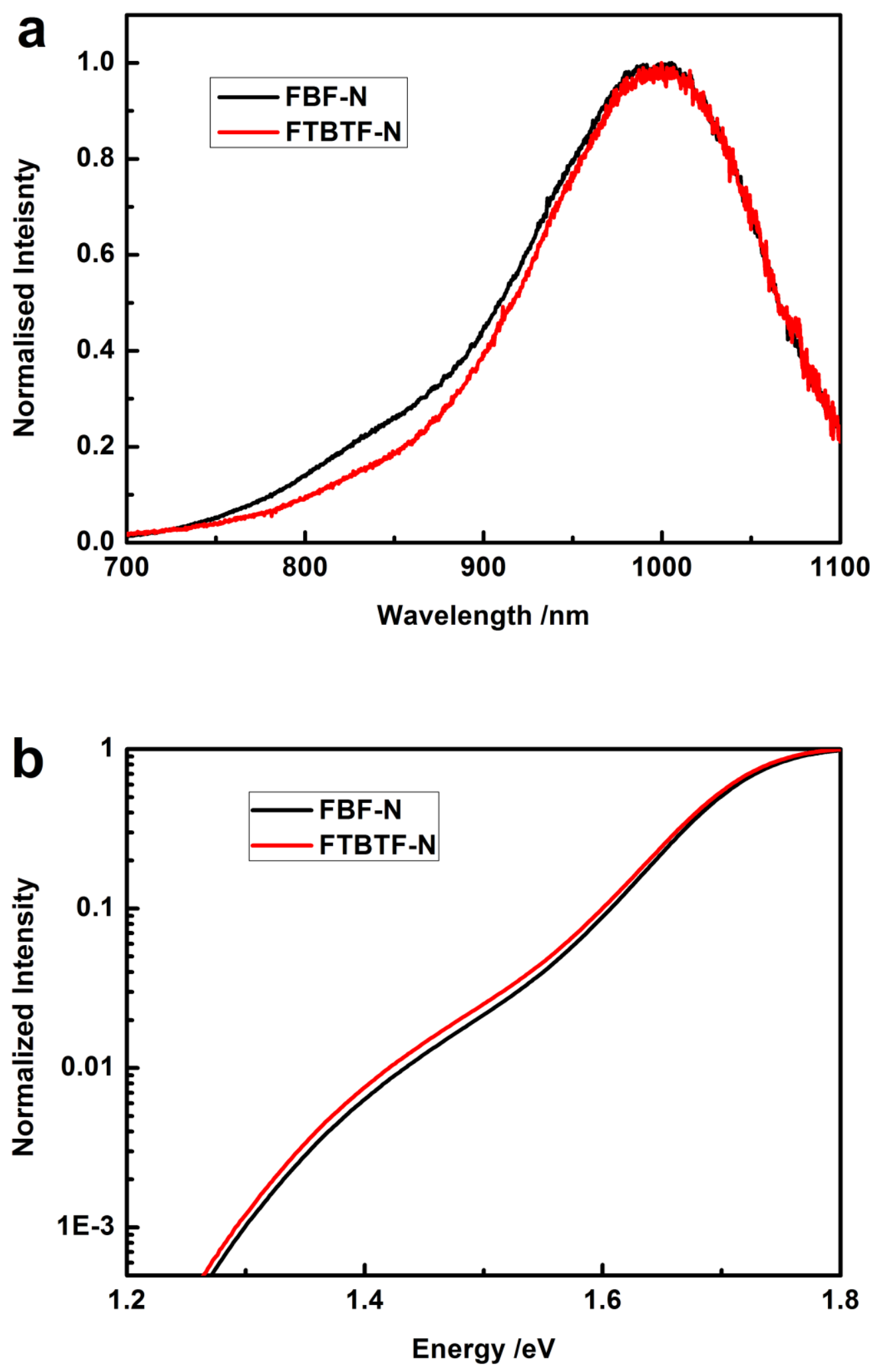

Figure 3. Normalized EL spectra (a) and normalized FTPS spectra (b) of inverted PSCs with FBF-N or FTBTF-N as the cathode interlayer. 


\section{WILEY-VCH}

Table 1. Photovoltaic performance of the inverted PSCs with and without the cathode interlayer.

\begin{tabular}{ccccc}
\hline Interface & $V_{o c}(\mathrm{~V})$ & $J_{s c}\left(\mathrm{~mA} / \mathrm{cm}^{2}\right)$ & $F F(\%)$ & PCE $(\%)$ \\
\hline Bare ITO & $0.25^{\mathrm{a}}\left(0.22^{\mathrm{b})}\right)$ & $14.02(12.97)$ & $33.46(30.89)$ & $1.18(0.92)$ \\
FBF-N & $0.75(0.75)$ & $15.50(15.67)$ & $68.22(66.62)$ & $7.97(7.85)$ \\
FTBTF-N & $0.74(0.74)$ & $17.23(17.30)$ & $72.11(69.85)$ & $9.22(8.93)$
\end{tabular}

${ }^{\text {a) }}$ Best device; b); ${ }^{\text {b) }}$ average values calculated over six devices. 


\section{WILEY-VCH}

Two alcohol-soluble organic molecules are synthesized and introduced into inverted organic solar cells as the cathode interlayer. A power conversion efficiency as high as $9.22 \%$ is obtained by using the more hydrophobic molecule FTBTF-N as the cathode interlayer. Morphological studies suggest that design of backbone can help to enhance $J_{s c}$ and $F F$.

organic solar cells, morphologies, structure-property relationships, cathode interlayers

W. Zhang, Y. Wu, Q. Bao, F. Gao*, J. Fang*

Morphological Control for Highly Efficient Inverted Polymer Solar Cells via Backbone Design of Cathode Interlayer Materials

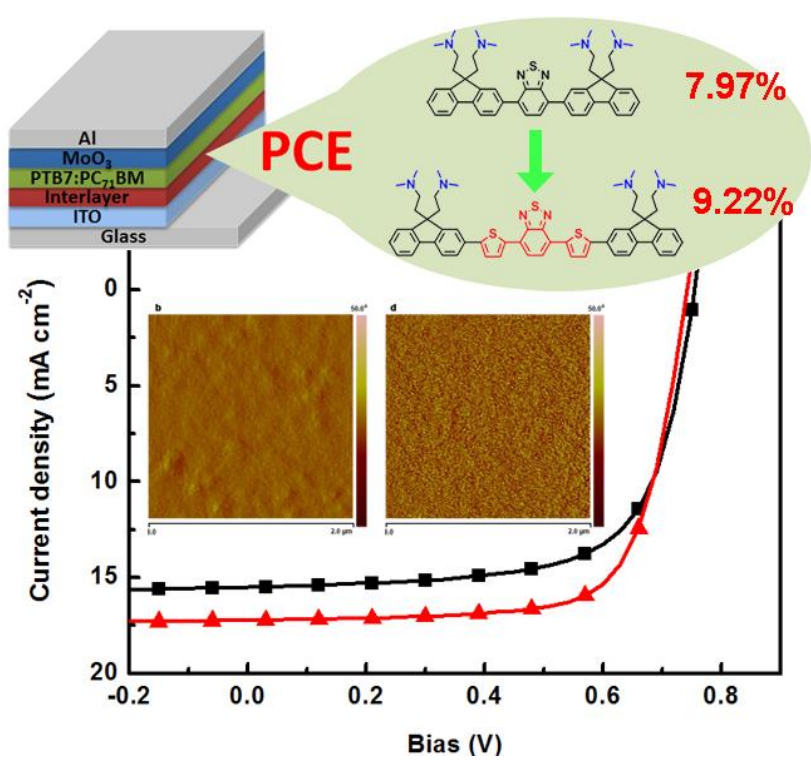

